\title{
Evaluation of Nitrate Reductase Assay for Detection of Multi-drug Resistant Mycobacterium tuberculosis among Patients at National Tuberculosis Reference Laboratory Zaria Nigeria
}

\author{
K. Mamuda ${ }^{1^{*}}$, O. S. Olonitola ${ }^{2}$, E. D. Jatau ${ }^{2}$ and E. Nicholas ${ }^{3}$ \\ ${ }^{1}$ National Tuberculosis and Leprosy Training Center, P.M.B.1089, Zaria, Kaduna, Nigeria. \\ ${ }^{2}$ Department of Microbiology, Faculty of Life Sciences, Ahmadu Bello University, Zaria, Nigeria. \\ ${ }^{3}$ Institute of Human Virology-Nigeria, National Tuberculosis and Leprosy Training Center, P.M.B.1089,
} Zaria, Kaduna, Nigeria.

Authors' contributions

This work was carried out in collaboration between all authors. Author KM designed the study. Authors KM and EN managed the literature searches. Authors EN and KM wrote the protocol. Author KM performed the statistical analysis. Authors OSO, EDJ and EN managed the analysis of the study. Authors KM and OSO wrote the first draft of the manuscript. All authors read and approved the final manuscript.

Article Information

DOI: 10.9734/JAMPS/2017/33705

Editor(s):

(1) Xiao-Xin Yan, Department of Anatomy \& Neurobiology, Central South University Xiangya School of Medicine (CSU-XYSM), Changsha, Hunan 410013, China. Reviewers:

(1) Germano Manuel Pires, Ministry of Health Mozambique, Mozambique.

(2) Abhishek Kumar, Nitte University, India. Complete Peer review History: http://www.sciencedomain.org/review-history/19611

Original Research Article

Received 26 $6^{\text {th }}$ April 2017

Accepted $18^{\text {th }}$ May 2017

Published $19^{\text {th }}$ June 2017

\section{ABSTRACT}

Aims: To evaluate nitrate reductase assay for detection of multi-drug resistant Mycobacterium tuberculosis among patients at National Tuberculosis Reference Laboratory Zaria Nigeria.

Study Design: Hospital based cross sectional study

Place and Duration of Study: National Tuberculosis Reference Laboratory Zaria Nigeria from December 2015 to June 2016.

Methodology: A total of 437 re-treatment patients' samples were screened for Acid Fast Bacilli (AFB), 72 were smear positive. Out of 72 smears positive, 62 were culture positive, using 
Lowenstein Jensen medium, 57 were found to be Mycobacterium tuberculosis complex (MTBC) using immunochromatographic test. In this study the susceptibility of $57 \mathrm{MTBC}$ isolates to isoniazid $(\mathrm{INH})$, rifampicin (RIF), streptomycin (STR) and Ethambutol (EMB) was determined by Lowenstein Jensen proportion method (LJPM) and Nitrate Reductase Assay (NRA)

Results: The sensitivity and specificity of NRA compared to that of LJPM were observed to be $98 \%$ and $89 \%, 98 \%$ and $92 \%, 64 \%$ and $80 \%, 68 \%$ and $77 \%$ for RIF, INH, STR, and EMB respectively. Positive predictive values were $91 \%, 93 \%, 87 \%$ and $83 \%$ for RIF, INH, STR and EMB respectively. Negative predictive values were $80 \%, 92 \%, 67 \%$ and $90 \%$ for RIF, INH, STR and EMB respectively .Overall, the sensitivity, specificity ,positive predictive value and negative predictive value of NRA in detecting MDR-TB were $90 \%, 82 \%, 85 \%$ and $73 \%$ respectively. Good agreement was found in all the tests with $\mathrm{k}$ values of $0.63,0.61,0.61$ and 0.62 for RIF, INH, EMB and MDR-TB respectively only STR shows moderate agreements with $\mathrm{k}$ value of 0.59 .

Conclusion: In the emergence of MDR-TB, the NRA may be of great importance due to its higher sensitivity and specificity for the rapid detection of rifampicin and isoniazid resistance, the two most important drugs for tuberculosis treatment. On the basis of our findings, NRA has the potential to be a useful tool for accurate detection of MDR-TB in the study area.

Keywords: Multidrug resistant TB; nitrate reductase assay; LJ proportion method; drug susceptibility testing.

\section{INTRODUCTION}

Tuberculosis (TB) is causing more deaths worldwide than any other infectious disease. The World Health Organization (WHO) estimated that in 2011, there were 8.7 million cases of TB, with nearly 1 million deaths among HIV-negative cases and 0.43 million deaths associated with HIV infection [1]. Case detection of all forms stood at $51 \%$. The mortality rate for all forms of TB remains 27 per 100,000 populations $(46,000$ deaths per year) [1]. Based on 2012 survey Nigeria ranked $4^{\text {th }}$ among the highest tuberculosis burden countries in the world and first in Africa. The point estimates of TB prevalence rates were 318 and 524 per 100,000 population (15 years and above) respectively [2].

Multidrug-resistant TB (MDR-TB) is TB that is resistant to atleast, rifampicin (RIF) and isoniazid (INH) the two most important first-line drugs with or without other first line drugs. In Nigeria, the national MDR-TB survey showed a prevalence of $2.9 \%$ among new cases and $14.3 \%$ for retreatment cases [1]. Currently there are 2 national Tuberculosis reference laboratories and 6 zonal Tuberculosis reference laboratories for culture which generally use Lowenstein-Jensen (LJ) medium with limited use of liquid cultures.

Indirect susceptibility testing on LJ medium is the most common method for detection of TB drug resistance in Africa. There is an urgent demand for early and proper detection of MDR and XDRTB cases for the effective management and control of TB. In recent years, a multitude of techniques for rapid DST has been designed and evaluated .However, the investment and recurrent costs is an obstacle for the broad implementation of these techniques in resourcelimited settings (RLSs) of Africa [3].

Global efforts for TB control, especially in resource limited settings, are being challenged by the lack of rapid, reliable and inexpensive techniques for the detection of $M$. tuberculosis. Results from the conventional culture detection methods come too late to influence a timely decision on patient management. Early detection is the key in both patient management and controlling transmission of $M$. tuberculosis. Liquid media systems such as the Mycobacterial Growth Indicator Tube (MGIT), are more rapid and detect more mycobacterial isolates than LJ [4]. The use of liquid systems the MGIT 960, has improved TAT to about 25-45 days. Liquid culture systems require expensive equipment and high biosafety level structures with constant electricity [5]. The traditional method for detection of MDR TB with indirect susceptibility testing, involving isolation of the bacterium followed by drug susceptibility testing (DST), has a long turnaround time (TAT) of 10 to 12 weeks [6]. Molecular tools such as Line Probe Assay (LPA) have been in use in Nigeria, but are expensive to run and require constant electricity. Therefore, faster, inexpensive and reliable tests are urgently needed [7].

Nitrate Reductase Assay (NRA) is a simple colorimetric method that requires basic TB culture equipment with short turnaround 
time.NRA is simple, inexpensive, reliable and reproducible test. Therefore, rapid, affordable, accurate and easy to use test for MDR-TB in Nigeria remains a priority. The aim of this study was to evaluate nitrate Reductase assay for detection of multi-drug resistant Tuberculosis compared to LJ proportion method, in Nigeria.

\section{MATERIALS AND METHODS}

\subsection{Settings}

The NTBLTC is the largest TB referral center in northern Nigeria; NTBLTC serves as the National Training Center for community health workers and laboratory health personnel's involved with Diagnosis of tuberculosis and treatment at the Peripheral, State and Zonal levels. This center is one of the two National TB reference laboratories that is equipped with TB biosafety level 3 (BSL-3) and TB molecular diagnostic laboratories.

\subsection{Sample Collection}

A total of 437 re-treatment patients samples were collected and screened for Acid Fast Bacilli (AFB).Positive smears were culture on Lowenstein Jensen Media while culture positive samples were first identified for MTBC using immunochromatographic test kits.

\subsection{Preparation of Lowenstein- Jensen Medium with Drugs}

Isoniazid (INH), Streptomycin (STR), Rifampicin (RIF) and Ethambutol (EMB) were obtained as powder from Sigma Aldrich (Bornem, Belgium). Each drug was prepared at a concentration of 10 $\mathrm{mg} / \mathrm{ml}$ in sterile distilled water with the exception of RIF, which was dissolved in dimethylsulphuroxide (DMSO). Stock solutions were filtered $(0.45 \mu \mathrm{m})$ sterilized and stored at $70{ }^{\circ} \mathrm{C}$ and used within six months. Different concentration of $0.2 \mu \mathrm{g} / \mathrm{ml} \mathrm{INH}, 2 \mu \mathrm{g} / \mathrm{ml} \mathrm{EMB}, 40$ $\mu \mathrm{g} / \mathrm{ml} \mathrm{RIF}$ and $4 \mu \mathrm{g} / \mathrm{ml}$ STR, were incorporated into LJ medium and then inspissated at $85^{\circ} \mathrm{C}$ for 45 minutes. After preparation, the media were incubated for 48 hours at room temperature for sterility check before use [8].

\subsection{Preparation of Lowenstein- Jensen Medium with $\mathrm{KNO}_{3}$ and Drugs}

Two grams of potassium nitrate were dissolved in $10 \mathrm{mls}$ of sterile distilled water. And $1 \mathrm{ml}$ of prepared $\mathrm{KNO}_{3}$ was added to $200 \mathrm{ml}$ of the drug containing and drug free LJ medium, dissolved by stirring, aliquoted and then inspissated for 45 minutes at $85^{\circ} \mathrm{C}$. After inspissations, the media was incubated for 48 hours at $37^{\circ} \mathrm{C}$ for sterility check before use. For NRA, the tubes containing $\mathrm{LJ}$ with antibiotics and $\mathrm{KNO}_{3}$ were used for the test while $\mathrm{LJ}$ with $\mathrm{KNO}_{3}$ were used as a control [8].

\subsection{Drug Susceptibility Testing by Proportion Method}

The Indirect PM (IPM) was performed on LJ medium with the same recommended critical concentrations of antibiotics as mentioned previously. The culture of $M$. tuberculosis strain was harvested and suspended in tube containing sterile distilled water with 5-7 sterile glass beads then vortexed for about 30 seconds to homogenise the bacterial suspension and then allowed to stand for 15-30minutes for large aggrevates of bacteria to settle and turbidity of bacterial suspension was adjusted to match the McFarland turbidity No. 1 . The original bacterial suspension was further diluted to $10^{-1}, 10^{-2}, 10^{-3}$ and $10^{-4}$. The tubes were arranged in order of $C_{1}$, $\mathrm{C}_{2}, \mathrm{C}_{3}, \mathrm{~S}, \mathrm{I}, \mathrm{R}$ and $\mathrm{E}$ representing $10^{-2}, 10^{-3} 10^{-4}$, streptomycin, Isoniazid, Rifampicin and Ethambutol respectively.

One in hundred dilutions $\left(10^{-2}\right)$ was inoculated into control $\left(\mathrm{C}_{1}\right)$ and four (4) drugs (S I R E), $10^{-3}$ dilution was inoculated into $\mathrm{C}_{2}$ and $10^{-4}$ was inoculated into $\mathrm{C}_{3}$ and then all tubes were incubated at $37^{\circ} \mathrm{C}$ for six weeks. Growth was recorded at 28 days and at 42 days as follows: $3+$ for confluent growth, 2+ for more than 100 colonies, and 1-100 actual numbers of colonies. Susceptibility or resistance was recorded when the proportion of bacteria in drug-containing medium to that of drug free medium is $<1$ or $\geq 1$ respectively. $M$. tuberculosis reference strains H37Rv (ATCC 27294) sensitive to first-line antituberculosis drugs, RIF-resistant (ATCC 35838), INH-resistant (ATCC 35822), EMB-resistant (ATCC 35837) and STR-resistant (ATCC 35820) were used as susceptible and resistant controls. All strains were sub cultured in LowensteinJensen (LJ) medium for 4 weeks before being studied [8].

\subsection{Drug Susceptibility Testing by Nitrate Reductase Assay}

Bacterial suspensions was made from a culture of MTB Isolates by dispensing a loopful of bacteria in $0.5 \mathrm{~mL}$ of sterile distilled water in 
$15 \mathrm{~mL}$ falcon tube containing a few $3-\mathrm{mm}$ diameter glass beads and vortexed to obtain a uniform solution., approximately $2.5 \mathrm{~mL}$ of sterile distilled water was added. The inoculum turbidity was adjusted to a McFarland tube no. 1 and diluted 1: 10 in sterile distilled water. Undiluted suspension $(200 \mu \mathrm{I})$ was inoculated into tubes of $\mathrm{LJ}$ medium with $\mathrm{KNO}_{3}(1 \mathrm{mg} / \mathrm{ml})$ containing each of the drugs at the concentrations described above and $200 \mu \mathrm{I}$ of the $1: 10$ dilution was inoculated into three control tubes without antibiotics. The assay was developed with reagent $\operatorname{mix}(50 \%$ conc. $\mathrm{HCl}, 0.2 \%$ sulfanilamide and $0.1 \% \quad \mathrm{~N}$-(1-naphthyl) ethylene diamine dihydrochloride at ratio of $1: 2: 2$ ) after 7 days of incubation at $37^{\circ} \mathrm{C}$, a $0.5 \mathrm{ml}$ of reagent mix was added to one control tube. When the clear reagent mixture turned pink, the drug-containing tubes were subsequently developed. When there was no colour change, the tubes were reincubated and the procedure repeated on days 10 and 14. An isolate was considered resistant if the drug-containing tube produced a colour change that was more intense than the drug-free tube. M. tuberculosis reference strains $\mathrm{H} 37 \mathrm{Rv}$ (ATCC 27294) sensitive to first-line antituberculosis drugs, RIF-resistant (ATCC 35838), INH-resistant (ATCC 35822), EMB-resistant (ATCC 35837) and STR-resistant (ATCC 35820) were used as susceptible and resistant controls. All strains were sub cultured in LowensteinJensen (LJ) medium for 4 weeks before being studied [9].

\section{RESULTS AND DISCUSSION}

Drug susceptibility testing was performed on NRA to first line drugs on which fifty seven (57) confirmed isolates, showed highest resistance as against rifampicin $(82.5 \%)$ followed by isoniazid resistance (77.2\%). Streptomycin and ethambutol resistances were $65 \%$ and $51 \%$ respectively. Whereas ethambutol had highest number of susceptible isolates of $49 \%$, streptomycin 35\%, isoniazid $22.8 \%$ and rifampicin (17.5\%) with lowest susceptible was recorded as shown in Table 1.

Multi-drug resistant tuberculosis is most difficult and expensive for management and treatment during the recent years is an increasing. Rapid detection of MDR strains is therefore needed to stop their spread in the population. Current methods for DST of MTB are either costly or very slow. So, a cost-effective and rapid drugsusceptibility method is required to guide the treatment of TB. NRA result of Bwanga et al. [10] in Uganda NRA was suggest that NRA is the most sensitive, specific, and cheapest test for MDR-TB in low income settings. Rapid detection of MDR-TB and thereby rapid drug susceptibility testing (DST) to first line drugs, are critically important to the successful control of TB [11].

The results of drug susceptibility testing by NRA indicate that the resistance rate was higher as against of isoniazid and rifampicin, which are the most important first line drugs used for the treatment of tuberculosis. This could be as a result of overuse of these drugs for the treatment of TB. This is in agreement with the work of Mahadev et al. [12] and lqbal et al. [13] who reported higher resistance to rifampicin and isoniazid.

The sensitivity and specificity of NRA compared to that of LJPM were observed to be $98 \%$ and $89 \%, 98 \%$ and $92 \%, 64 \%$ and $80 \%, 68 \%$ and $77 \%$ for RIF, INH, STR, and EMB respectively. Positive predictive values were 91\%, 93\%, 87\% and $83 \%$ for RIF, INH, STR and EMB respectively. Negative predictive values were $80 \%, 92 \%, 67 \%$ and $90 \%$ for RIF, INH, STR and EMB respectively. Overall, the sensitivity, specificity, positive predictive value and negative predictive value of NRA in detecting MDR-TB were $90 \%, 82 \%, 85 \%$ and $73 \%$ respectively. Good agreement was found in all the tests with $\mathrm{K}$ values of $0.63,0.61,0.61$ and 0.62 for RIF, INH, EMB and MDR-TB respectively only STR shows moderate agreements with $k$ value of 0.59 (Table 2).

When the results of NRA and LJ PM were compared, higher agreement was observed for RIF and INH, however there were disagreements in relation to the antibiotics EMB and STR. The factors which led to lower specificity and sensitivity values of LJ could be as a result of degradation of the antibiotics in LJ medium. The higher rate of detection of RIF resistance by NRA method is the reason for the use of NRA for the screening of MDR strains, as resistance to RIF is a marker for MDR detection. Most studies have shown high sensitivity and specificity values for $\mathrm{INH}$ and RIF, the two most important drugs used for TB treatment $[9,14,15]$.

In a multicentre study, the NRA was evaluated in different settings to determine the susceptibility of $M$. tuberculosis to first-line anti-tuberculosis drugs. Higher accuracy of $96.6 \%$ was observed for INH and RIF, while that for STR and EMB were lower (85.3\%) [16]. Percentage agreement of $98.3 \%, 98.3 \%, 90.8 \%$ and $93.3 \%$ by NRA 
Table 1. Drug susceptibility of Mycobacterium tuberculosis complex by nitrate reductase assay

\begin{tabular}{lcccc}
\hline Drugs & Streptomycin & Isoniazid & Rifampicin & Ethambutol \\
\hline Resistance & $37(65 \%)$ & $44(77.2 \%)$ & $47(82.5 \%)$ & $29(59 \%)$ \\
Susceptible & $20(35 \%)$ & $13(22.8 \%)$ & $10(17.5 \%)$ & $28(49 \%)$ \\
\hline \multicolumn{4}{c}{ Key=MDR-TB $=$ multidrug resistant tuberculosis, NRA = nitrate reductase assay, LJPM = Lowenstein Jensen } \\
\multicolumn{4}{c}{ proportion Method }
\end{tabular}

Table 2. Evaluation of drug susceptibility testing of Mycobacterium tuberculosis using nitrate reductase assay and LJ proportion method

\begin{tabular}{llllll}
\hline Drugs & Sensitivity (\%) & Specificity (\%) & PPV (\%) & NPV (\%) & k-statistic \\
\hline Streptomycin & 64 & 80 & 87 & 67 & 0.59 \\
Isoniazid & 98 & 92 & 93 & 92 & 0.61 \\
Rifampicin & 98 & 89 & 91 & 80 & 0.63 \\
Ethambutol & 68 & 77 & 83 & 90 & 0.61 \\
MDR-TB & 90 & 82 & 85 & 73 & 0.62 \\
\hline \multicolumn{5}{r}{ Key: PPV = positive predictive value, NPV = negative predictive value, $k=$ kappa }
\end{tabular}

were observed for INH, RIF, STR and EMB respectively by Shikama et al. [17]. However, similar lower sensitivity and specificity for STR and EMB have also been reported by Lemus et al. [18] and Montoro et al. [19] as also reported in this study.

Lemus et al. [18] in India also observed sensitivity of $91.7 \%, 96.5 \%, 88.0 \%$ and $93.9 \%$ for INH, STR, EMB and RIF, respectively, and then specificity was higher than $99.1 \%$ for all of the drugs. While in this study higher sensitivity for $\mathrm{INH}$ and RIF was observed to be $98 \%$ and this could be as the result of the patients categories used for the study. Visalakshi et al. [20] observed sensitivity and specificity of the direct NRA and indirect LJPM to be $94 \%$ and $98 \%$, and $100 \%$ and $98 \%$ for RIF and INH respectively which is similar to the result obtained in the study. Moreover, Bwanga et al. [10] in Uganda stated $98 \%$ sensitivity and specificity of NRA for RIF and $98 \%$ sensitivity and $94 \%$ specificity of NRA for INH. In another study of Gupta et al., [21] sensitivity of NRA was $98.4 \%, 97 \% 88.5 \%$ and $94.5 \%$ for RIF, INH, STR and EMB with $99 \%$ agreement between the results of NRA and LJPM for INH and RIF while this study have reported lower sensitivity for STR and EMB.

The higher detection rate of MDR-TB by NRA could be as a result of borderline that exhibit color change lower than that obtained in the control tube and therefore this borderline isolate are frequently associated with problems of interpretation of DST by NRA. Bacterial contamination may cause false positive results and low inoculation in the media could also result in false positive result [22]. More than half of
MDR-TB isolates were resistant to all four firstline anti tuberculosis drugs tested. Re-treatment cases were significantly more likely to have MDR-TB than non-MDR-TB. Previous studies have shown that resistance to rifampicin is a significant predictor of resistance to isoniazid and streptomycin; isolates resistant to rifampicin were also resistant to isoniazid and streptomycin [23].

\section{CONCLUSION}

In the context of emergence of MDR-TB, the NRA may be of great importance due to its higher sensitivity and specificity for the rapid detection of rifampicin and isoniazid resistance, the two most important drugs for tuberculosis treatment. On the basis of the findings, NRA has the potential to be a useful tool for accurate detection of MDR-TB in the study area.

\section{CONSENT}

All authors declare that 'written informed consent was obtained from the patient (or other approved parties) for publication of this case report and accompanying images.

\section{ETHICAL APPROVAL}

All authors hereby declare that all experiments have been examined and approved by the appropriate ethics committee and have therefore been performed in accordance with the ethical standards.

\section{COMPETING INTERESTS}

Authors have declared that no competing interests exist. 


\section{REFERENCES}

1. World Health Organization. Global tuberculosis report 2012. Geneva, Switzerland: World Health Organization; 2012.

Available:http://apps.who.int/iris/bitstream/ 10665/75938/1/9789241564502 eng.pdf

2. World Health Organization. Report FIRST National TB Prevalence Survey. 2012;V(1): 20-25.

3. Franzblau SG, Witzig RS, McLaughlin JC, Torres P, Madico G, Hernandez A. Rapid, low-technology MIC determination with clinical Mycobacterium tuberculosis by using the micro plate Alamar blue assay. Journal of Clinical Microbiology. 1998; 36:362-366.

4. Goloubeva V, Lecocq M, Lassowsky P, Matthys F, Portaels F, Bastian I. Evaluation of mycobacteria growth indicator tube for direct and indirect drug susceptibility testing of Mycobacterium tuberculosis from respiratory specimens in a Siberian prison hospital. Journal of Clinical Microbiology. 2001;39:1501-1505.

5. Bouyer WN, Yorke R, Lee HI, Woods GL. Comparison of the BACTEC MGIT 960 and ESP culture system II for growth and detection of Mycobacteria. Journal of Clinical Microbiology. 2000;38:4167-4170.

6. Golyshevskaia $\mathrm{VI}$, Korneev AA, Chernousova LN, Selina LG, Kazarova TA, Grishina TD. New microbiological techniques in diagnosis of tuberculosis. Problemy Tuberkuleza. 1996;6:22-25.

7. Moore DA, Evans CA, Gilman $\mathrm{RH}$, Caviedes L, Coronel J. Microscopic observation drug susceptibility assay for the diagnosis of TB. New England Journal of Medicine. 2006;355(15):1539-1550.

8. Canetti G, Froman S, Grosset J, Hauduroy $\mathrm{P}$, Langerova M, Mahler HT. Mycobacteria: Laboratory methods for testing drug sensitivity and resistance. Bulletin of World Health Organization. 1963;29:565578.

9. Angeby KA, Klintz L, Hoffner SE. Rapid and inexpensive drug susceptibility testing of Mycobacterium tuberculosis with a nitrate reductase assay. Journal of Clinical Microbiology. 2002;40(2):553-555.

10. Bwanga F, Hoffner S, Haile M, Joloba ML. Direct susceptibility testing for multi drug resistant tuberculosis: A meta-analysis. Biomedical of Infectious Disease. 2009; 9(1):15-20.
11. World Health Organization. Policy statement: Automated real-time nucleic acid amplification technology for rapid and simultaneous detection of tuberculosis and rifampicinresistance:

XpertMTB/ RIFsystem; 2011b.

Available:who.int/publications/2011/97892 41501545 eng.pdf

(Accessed 7 June 2012)

12. Mahadev B, Kumar P, Agarwal SP, Chauhan LS, Srikantaramu N. Surveillance of drug resistance to antituberculosis drugs in districts of Hooghly in west Bengal and Mayurbhanj in Orissa. Indian Journal for Tuberculosis. 2004;48:129-134

13. Iqbal R, Shabbir I, Khan SU, Saleem S, Munir K. Multidrug resistance tuberculosis in Lahore. Pakistan Journal of Medical Research. 2008;47(1):26-28.

14. Lemus D, Martin A, Montoro E, Portaels F, Palomino JC. Rapid alternative methods for detection of rifampicin resistance in Mycobacterium tuberculosis. Journal Antimicrobial Chemotherapy. 2004; 54:130-133.

15. Simboli N, Takiff H, McNerney R, Lo' pez B, Martin A, Palomino JC, Barrera L, Ritacco V. In-house phage amplification assay is a sound alternative for detecting rifampin-resistant Mycobacterium tuberculosis in low-resource settings. Antimicrobial Agents Chemotherapy. 2005; 49:425-427.

16. Martin A, Montoro E, Lemus D. Multicenter evaluation of the nitrate reductase assay for drug resistance detection of Mycobacterium tuberculosis. Journal of Microbiological Methods. 2005;63:145150.

17. Shikama Mde L, Silva RR, Martins MC. Rapid detection of resistant tuberculosis by nitrate reductase assay performed in three settings in Brazilian. Journal of Antimicrobial Agents and Chemotherapy. 2009;64:794-96.

18. Lemus D, Montoro E, Echemendia M, Martin A, Portaels F, Palomino JC. Nitrate reductase assay for detection of drug resistance in Mycobacterium tuberculosis: Simple and inexpensive method for lowresource laboratories. Journal for Medical Microbiology. 2006;55:861-863.

19. Montoro E, Lemus D, Echemendía M, Martin A, Portaels F, Palomino JC. Comparative evaluation of the nitrate reduction assay, the MTT test and the resazurin microtiter assay for drug 
susceptibility testing of clinical isolates of Mycobacterium tuberculosis. Journal of Antimicrobial Chemotherapy. 2005; 55:500-505.

20. Visalakshi P, Meharwal SK, Myneedu VP, Behera D. Evaluation of direct method of drug susceptibility testing of Mycobacterium tuberculosis to rifampicin and isoniazid by nitrate reductase assay in a national reference laboratory. Diagnosis of Microbiology Infectious Disease. 2010; 66:148-52.

21. Gupta A, Ranjan Sen, Mohan Mohapatra $\mathrm{T}$, Anupurba S. Evaluation of the performance of nitrate reductase assay for rapid drug-susceptibility testing of
Mycobacterium tuberculosis in North India. International Centre for Diarrhoeal Disease Research, Bangladesh. 2011;1:20-25.

22. Kent P, Kubica G. Public health mycobacteriology: A guide for the level III laboratory, U.S. Department of Health and Human Services. Atlanta, Ga. 1985;96103.

23. Mdivani N, Zangaladze E, Volkova N, Kourbatova E, Jibuti T, Shubladze N, Kutateladze T, Khechinashvili G, del Rio C, Salakaia A, Blumberg HM. High prevalence of multidrug-resistant tuberculosis in Georgia. International Journal for Infectious Diseases. 2008; 12(6):635-644.

(c) 2017 Mamuda et al.; This is an Open Access article distributed under the terms of the Creative Commons Attribution License (http://creativecommons.org/licenses/by/4.0), which permits unrestricted use, distribution, and reproduction in any medium, provided the original work is properly cited.

Peer-review history:

The peer review history for this paper can be accessed here: http://sciencedomain.org/review-history/19611 